\title{
Bacteraemia in Intensive Care Unit: Clinical, Bacteriological, and Prognostic Prospective Study
}

\author{
Zineb Lachhab, ${ }^{1,2}$ Mohammed Frikh, ${ }^{1,2}$ Adil Maleb, ${ }^{2,3}$ Jalal Kasouati, ${ }^{4}$ \\ Nouafal Doghmi, ${ }^{5}$ Yassine Ben Lahlou, ${ }^{1,2}$ Bouchra Belefquih, ${ }^{1,2}$ Abdelhay Lemnouer, ${ }^{1,2}$ \\ and Mostafa Elouennass ${ }^{1,2}$ \\ ${ }^{1}$ Bacteriology Department, Mohammed V Military Teaching Hospital, Rabat, Morocco \\ ${ }^{2}$ Research Team, Bacterial Epidemiology and Resistance, Mohammed V University, Faculty of Medicine and Pharmacy, Rabat, Morocco \\ ${ }^{3}$ Microbiology Department, Mohammed VI University Hospital, Oujda, Morocco \\ ${ }^{4}$ Epidemiology Department, Mohammed V Military Teaching Hospital, Rabat, Morocco \\ ${ }^{5}$ Medical Reanimation Department, Mohammed V Military Teaching Hospital, Rabat, Morocco
}

Correspondence should be addressed to Adil Maleb; maleb.adil@gmail.com

Received 29 December 2016; Revised 6 March 2017; Accepted 13 March 2017; Published 19 March 2017

Academic Editor: Jorge Garbino

Copyright (c) 2017 Zineb Lachhab et al. This is an open access article distributed under the Creative Commons Attribution License, which permits unrestricted use, distribution, and reproduction in any medium, provided the original work is properly cited.

Objectives. We conducted a one-year observational study from December 2012 to November 2013 to describe the epidemiology of bacteraemia in intensive care units (ICU) of Mohammed V Military Teaching Hospital of Rabat (Morocco). Methods. The study consisted of monitoring all blood cultures coming from intensive care units and studying the bacteriological profile of positive blood cultures as well as their clinical significance. Results. During this period, a total of 46 episodes of bacteraemia occurred, which corresponds to a rate of $15,4 / 1000$ patients. The rate of nosocomial infections was $97 \%$ versus $3 \%$ for community infections. The most common source of bacteraemia was the lungs in 33\%, but no source was identified in $52 \%$ of the episodes. Gram negative organisms were isolated in $83,6 \%$ of the cases with Acinetobacter baumannii being the most frequent. Antibiotic resistance was very high with $42,5 \%$ of extended-spectrum beta-lactamases (ESBLs) in Enterobacteriaceae and $100 \%$ of carbapenemase in Acinetobacter baumannii. The antibiotherapy introduced in the first 24 hours was adequate in $72 \%$ of the cases. Conclusions. Bloodstream infections in ICU occur most often in patients over 55 years, with hypertension and diabetes. The bacteria involved are mainly Gram negative bacteria multiresistant to antibiotics. Early administration of antibiotics significantly reduces patients mortality.

\section{Background}

Bacteraemia continues to be an important cause of morbidity and mortality despite the availability of potent antimicrobial agents and sophisticated diagnostic means [1]. There is a continuous rise worldwide in bacteraemia incidence, which is related to many factors as the population demographic differences and risk factor distribution in regions [2].

Over the past 30 years, the incidence, etiology, and epidemiology of bacteraemia have changed with the evolution of medical care [3]. In developed countries, these parameters are well known through regular monitoring [4]. Thus, several studies have treated the etiologic agents most frequently responsible for these infections and improved the understanding of the risks and consequences associated with them [5]. In resource-limited countries, such as Morocco, the lack of sufficient data makes bacteraemia one of the daily worries of medical practice especially in intensive care units (ICU) where patients are predisposed to acquire them.

Bacteraemia is a major problem to manage. It is indeed necessary to find a compromise between the urgency of the treatment and the difficulty to pose a precise diagnosis within a short time [1]. In this context, it is important for the clinician to know the most frequently encountered bacterial species and their sensitivity to antibiotics to initiate prompt and effective empiric antibiotic therapy. As a part of the evolution of the bacterial ecology and the changes in the levels of resistance to antibiotics, periodic revaluation of the management of bacteraemic patients is necessary, in order to tailor for better treatment decisions [6]. 
The aim of this study is to determine the incidence of bacteraemia in ICU of our hospital, to establish the bacterial epidemiology and the susceptibility of the isolated strains to antibiotics.

\section{Methods}

This observational study was conducted prospectively at the medical and surgical ICUs of Mohammed V Military Teaching Hospital of Rabat, Morocco. The study was designed to include all sequentially encountered episodes of bacteraemia during the period December 2012-November 2013.

The study consisted of monitoring, from the bacteriology laboratory, all blood cultures coming from ICU. Aerobic and anaerobic blood culture vials were performed at the patient's bed by peripheral puncture or through a peripheral or central device newly installed. They are transported then to the bacteriology laboratory where they are incubated with agitation at $37^{\circ} \mathrm{C}$ in the system Bactec 9240 (Becton Dickinson).

We perform, from positive bottles, a transplanting on enriched medium and smear for Gram stain. Based on this direct examination and the front of a monomorphic appearance, biochemical identification and an antibiogram were made directly from the blood culture broth. The identification of species was based on the growth and the morphological and biochemical characteristics (API gallery, Biomérieux, Marcy-Star/France). The antimicrobial susceptibility testing of the isolates was performed by disk diffusion agar method, as recommended by the Committee of the Susceptibility of the French Society for Microbiology [7].

Each positive blood culture leads to a clinical survey by the author and the filling of an exploitation plug [8]. Concerning antibiotic treatment, we retained an average length of 10 days of treatment; and we noted the treatment received by the patient from day 0 of bacteraemia (the day of collection of blood culture) to day 10 . The cost of antimicrobial treatment per day per infected patient was calculated on the basis of the antimicrobial agent initiated on day 0 (including inappropriate treatment) and by multiplying the unit's price per dose and per the number of daily doses up to day 10 . The costs are calculated in Moroccan Dirham (MAD) and expressed in Euro $(\mathrm{EUR} / \mathrm{MAD}=10)$, on the basis of the 2012 price of antimicrobial agents provided by the military hospital's pharmacy.

Quantitative variables were expressed as mean +/- standard deviation. Chi square test was used to compare data between different groups; the results were considered significant if the $p$ value was less than 0.05 . Statistical analysis was carried out using the software package SPSS v10.0.

\section{Results}

From December 2012 to November 2013, 46 episodes of bacteraemia were recorded in 39 patients; the overall incidence was 20/1000 days of hospitalization and 15.4/1000 patients admitted at ICU. Table 1 shows the demographic and clinical data of patients. Most patients (97\%) had nosocomial bacteraemia and primary bacteraemia accounted for $26.7 \%$ of the cases. The most common source of bacteraemia was the lungs (33\%) but no source was identified in $52 \%$ of cases
(Table 2). There was $83.6 \%$ Gram negative bacilli (GNB) bacteraemia; overall, Acinetobacter baumannii was the most offending germ in bacteraemia and Klebsiella pneumoniae in contamination. Regarding yeasts, one germ out of the four isolated was responsible for bacteraemia; it was of Candida tropicalis. Commensal bacteria were responsible for contamination in all the cases (70.5\%) (Table 2).

Thirty-eight (69\%) of bacteraemic episodes were caused by antibiotic-resistant organisms. Among the GNB, all Pseudomonas spp. and Acinetobacter spp. were sensitive to colistin; however, a resistance to imipenem was observed among total isolates of Acinetobacter (13/13) in the context of the production of carbapenemase, while Pseudomonas aeruginosa resistance is emerging with a rate of $20 \%$. The rate of Enterobacteriaceae with ESBL in this study was $42.5 \%$ essentially present in Klebsiella pneumoniae with a rate of $44 \%$. Only $20 \%(1 / 5)$ of Staphylococcus aureus isolates were resistant to methicillin; and on the other hand, no Gram positive germ showed decreased susceptibility to glycopeptides (Table 3).

With regard to antibiotic therapy, we found that almost all patients (97.5\%) received a curative antibiotic therapy that has proven adequate in the first 24 hours of its introduction in $72 \%$ of the cases, but the rate of efficiency was limited to $37 \%$. This initial treatment was an association of antibiotics in $57 \%$ of the cases. The most frequent association was beta-lactam with aminoglycosides, while the most used drug in monotherapy was colistin and imipenem (21.4\%). The molecules used are indicated in Figure 1. Initial treatment lasted in $33 \%$ of the cases one day before being changed; the main reasons for this change were either being inadequate, being ineffective, or realization of deescalation. However, ulterior treatment was documented in 59\% of cases and remained probabilistic in $41 \%$ of cases. The antibiotic association rate was higher than the initial treatment, with a value of $82 \%$. The most common association was the beta-lactams and polymyxins, while imipenem was the most prescribed molecule (Figure 1). This treatment proved adequate in $93 \%$ of the cases and effective in $65 \%$ of the cases (Table 4 ).

Twenty-seven patients (69\%) died during their ICU stay. For 21 of them, the death was directly attributed to bacteraemia. Death rate in the population under study compared to all deaths in the ICU during the study period was $12.5 \%$. The delay between the occurrence of bacteraemia and death was on average of 8,4 days. Comparisons of demographic and clinical characteristics of survived and deceased patients are described in Table 5.

\section{Discussion}

The incidence of bacteraemia in our study was $15.4 / 1000$ patients. Several reasons can explain the discrepancy of the incidence rate between our study and the other international studies, such as the type of the studied population and blood culture rates $[9,10]$.

Our demographics data showed that bacteraemia affects mainly males and elderly population ( $>55$ years), in agreement with several studies $[1,11,12]$. The predominance of men appears without explanation, unlike the advanced age which is related to a deterioration of the immune system 
TABLE 1: Demographic and clinical data of the 39 patients involved in the study.

\begin{tabular}{|c|c|c|c|}
\hline \multicolumn{2}{|c|}{ Variable } & Number, mean $+/-$ SD & $\%$ \\
\hline \multirow{2}{*}{ Gender } & M & 24 & $62 \%$ \\
\hline & $\mathrm{F}$ & 15 & $38 \%$ \\
\hline \multicolumn{2}{|c|}{ Age $($ mean $+/-S D)$} & $59,1+/-7,6$ & - \\
\hline \multirow{7}{*}{ Age group } & $15-25$ & 2 & $5,1 \%$ \\
\hline & $25-35$ & 2 & $5,1 \%$ \\
\hline & $35-45$ & 2 & $5,1 \%$ \\
\hline & $45-55$ & 4 & $10,2 \%$ \\
\hline & $55-65$ & 14 & $35,9 \%$ \\
\hline & $65-75$ & 11 & $28,2 \%$ \\
\hline & $75-85$ & 4 & $10,2 \%$ \\
\hline \multirow{6}{*}{ Underlying illness } & Essential hypertension & 13 & $34,2 \%$ \\
\hline & Diabetes mellitus & 12 & $30,8 \%$ \\
\hline & Immunosuppression & 3 & $8,1 \%$ \\
\hline & Chronic renal disease & 2 & $5,4 \%$ \\
\hline & Ischemic heart disease & 4 & $10,4 \%$ \\
\hline & Others & 12 & $31,6 \%$ \\
\hline \multirow{3}{*}{ Severity } & Sepsis & 23 & $50 \%$ \\
\hline & Severe sepsis & 8 & $17,4 \%$ \\
\hline & Septic shock & 15 & $32,6 \%$ \\
\hline \multirow{3}{*}{ Scores of gravity } & GLASGOW II & $10,14+/-3,76$ & - \\
\hline & APACHE II & $18,95+/-3,76$ & - \\
\hline & Predicted mortality & $35,07+/-22,2$ & - \\
\hline & stay & $41+/-6,4$ & - \\
\hline \multirow{4}{*}{ Antecedents } & Recent antibiotic use & 13 & $33,3 \%$ \\
\hline & Recent hospitalization & 23 & $59 \%$ \\
\hline & Recent surgery & 14 & $34,5 \%$ \\
\hline & Recent corticosteroid therapy & 24 & $61,5 \%$ \\
\hline \multirow{2}{*}{ Outcome } & Clinical failure & 17 & $44 \%$ \\
\hline & Clinical success & 22 & $56,5 \%$ \\
\hline \multicolumn{2}{|c|}{ Bacteraemia acquisition time } & $16,2+/-20,27$ & - \\
\hline
\end{tabular}

exposing the elderly to a high risk of contracting infectious diseases including bacteraemia. Zhang et al. reported that catheterization, mechanical ventilation, and urinary catheter were considered as risk factors for bacteraemia acquisition [13]. Our study supported the correlation of this factors and the high incidence found, since almost all of our population has been exposed to such maneuvers.

In this study, we confirm the predominance of GNB bacteraemia since we found it at the rate of $83.6 \%$; this theory has also been found in a recently published international observational study providing information about bacteraemia from 1,156 ICU patients worldwide [14]. The most alarming GNB in our case was Acinetobacter baumannii with a frequency of $23.6 \%$; this result appears wary compared to those reported in the literature [15]. Acinetobacter baumannii is responsible for more and more severe infections and the incidence of bloodstream infections caused by this organism is constantly increasing [16]. A study conducted in the UK showed that the rate of bacteraemia caused by Acinetobacter spp. increased from 2 to 18 per 100,000 hospital days between 1998 and 2005. Resistance to carbapenems has increased during the same period from $0 \%$ to $55 \%$ [17]. The carbapenems are the antibiotics of choice for the treatment of serious infections due to multidrug-resistant Acinetobacter baumannii. Unfortunately, the number of isolates of Acinetobacter baumannii resistant to carbapenems has increased in the recent years, which is a big problem because the resistance to carbapenems limits the clinician's options for successful treatment and leads to increased mortality [17]. Indeed the Acinetobacter baumannii isolates in our study showed $100 \%$ resistance to imipenem. This rate is extremely higher than that reported in the literature [18]. Several studies have indicated that risk factors for carbapenem-resistant Acinetobacter baumannii acquisition were higher in longer hospital stays, invasive procedures, admission to ICU, and irrational use of carbapenems [19, 20].

Klebsiella pneumoniae was the second organism causing bacteraemia in our study with a rate of $18.2 \%$. This value is higher than that reported in other studies [1]. This germ presented a high resistance rate vis-a-vis the beta-lactams and aminoglycosides. ESBL rate in our study was very high compared to that reported in the study of Elouennass et al. [21] 
TABLE 2: Distribution of different isolates in relation to setting of source of bacteraemia.

\begin{tabular}{|c|c|c|c|c|c|c|c|c|}
\hline \multirow{2}{*}{ Families } & \multirow{2}{*}{ Bacteria } & \multirow{2}{*}{ Total (\%) } & \multirow{2}{*}{ Bacteraemia (\%) } & \multirow{2}{*}{ Contamination (\%) } & \multicolumn{4}{|c|}{ Source of bacteraemia } \\
\hline & & & & & UN & $\mathrm{P}$ & $\mathrm{U}$ & CVC \\
\hline \multirow{9}{*}{ Enterobacteriaceae } & E. aerogenes & $1(0,7 \%)$ & $1(100 \%)$ & $0(0 \%)$ & $0(0 \%)$ & $1(100 \%)$ & $0(0 \%)$ & $0(0 \%)$ \\
\hline & E. cloacae & $3(2 \%)$ & $2(67 \%)$ & $1(33 \%)$ & $1(50 \%)$ & $0(0 \%)$ & $0(0 \%)$ & $1(50 \%)$ \\
\hline & E. coli & $3(2 \%)$ & $2(67 \%)$ & $1(33 \%)$ & $1(50 \%)$ & $0(0 \%)$ & $1(50 \%)$ & $0(0 \%)$ \\
\hline & K. pneumoniae & $16(10,7 \%)$ & $10(62,5 \%)$ & $6(37,5)$ & $9(90 \%)$ & $0(0 \%)$ & $1(10 \%)$ & $0(0 \%)$ \\
\hline & M. morganii & $2(1,3)$ & $2(100 \%)$ & $0(0 \%)$ & $1(50 \%)$ & $0(0 \%)$ & $0(0 \%)$ & $1(50 \%)$ \\
\hline & P. mirabilis & $5(2,7)$ & $2(40 \%)$ & $3(60 \%)$ & $2(100 \%)$ & $0(0 \%)$ & $0(0 \%)$ & $0(0 \%)$ \\
\hline & P. stuartii & $2(1,3)$ & $1(50 \%)$ & $1(50 \%)$ & $1(100 \%)$ & $0(0 \%)$ & $0(0 \%)$ & $0(0 \%)$ \\
\hline & S. marcescens & $3(2 \%)$ & $2(67 \%)$ & $1(33 \%)$ & $2(100 \%)$ & $0(0 \%)$ & $0(0 \%)$ & $0(0 \%)$ \\
\hline & S. odorifera & $5(3,3)$ & $3(60 \%)$ & $2(40 \%)$ & $1(33 \%)$ & $2(67 \%)$ & $0(0 \%)$ & $0(0 \%)$ \\
\hline \multirow{5}{*}{$\begin{array}{l}\text { Nonfermentative Gram } \\
\text { negative bacilli } n=20\end{array}$} & A. baumannii & $18(12 \%)$ & $13(72 \%)$ & $5(28 \%)$ & $4(31 \%)$ & $7(54 \%)$ & $0(0 \%)$ & $2(15 \%)$ \\
\hline & P. aeruginosa & $10(6,7)$ & $5(50 \%)$ & $5(50 \%)$ & $3(60 \%)$ & $1(20 \%)$ & $1(20 \%)$ & $0(0 \%)$ \\
\hline & Chryseobacterium sp. & $1(0,7)$ & $0(0 \%)$ & $1(100 \%)$ & I & I & I & I \\
\hline & Sphingomonas sp. & $1(0,7)$ & $1(100 \%)$ & $0(0 \%)$ & $1(100 \%)$ & $0(0 \%)$ & $0(0 \%)$ & $0(0 \%)$ \\
\hline & S. maltophilia & $2(1,3)$ & $1(50 \%)$ & $1(50 \%)$ & $1(100 \%)$ & $0(0 \%)$ & $0(0 \%)$ & $0(0 \%)$ \\
\hline \multirow{2}{*}{ Staphylococci $n=65(44 \%)$} & S. aureus & $5(3,3 \%)$ & $5(100 \%)$ & $0(0 \%)$ & $2(40 \%)$ & $3(50 \%)$ & $0(0 \%)$ & $0(0 \%)$ \\
\hline & CNS & $60(40 \%)$ & $0(0 \%)$ & $40(100 \%)$ & 1 & 1 & 1 & 1 \\
\hline \multirow{2}{*}{ Streptococci $n=6(4 \%)$} & E. faecalis & $5(3,3 \%)$ & $2(40 \%)$ & $3(60 \%)$ & $2(100 \%)$ & $0(0 \%)$ & $0(0 \%)$ & $0(0 \%)$ \\
\hline & S. mitis & $1(0,7 \%)$ & $1(100 \%)$ & $0(0 \%)$ & $1(100 \%)$ & $0(0 \%)$ & $0(0 \%)$ & $0(0 \%)$ \\
\hline \multirow{2}{*}{$\begin{array}{l}\text { Gram positive bacilli in } n=2 \\
(1.3 \%)\end{array}$} & Bacillus spp. & $1(0,7 \%)$ & $0(0 \%)$ & $1(100 \%)$ & 1 & I & I & 1 \\
\hline & Corynebacterium sp. & $1(0,7 \%)$ & $0(0 \%)$ & $1(100 \%)$ & l & l & l & l \\
\hline BGN demanding $n=1(0.7 \%)$ & H. influenza & $1(0,7 \%)$ & $1(100 \%)$ & $0(0 \%)$ & $0(0 \%)$ & $1(100 \%)$ & $0(0 \%)$ & $0(0 \%)$ \\
\hline \multirow{2}{*}{ Yeasts $n=4(2.7 \%)$} & C. albicans & $1(0,7 \%)$ & $0(0 \%)$ & $1(100 \%)$ & 1 & I & 1 & 1 \\
\hline & C. non-albicans & $3(2 \%)$ & $1(33 \%)$ & $2(67 \%)$ & $0(0 \%)$ & $0(0 \%)$ & $1(100 \%)$ & $0(0 \%)$ \\
\hline
\end{tabular}

UN: unknown, P: pulmonary, U: urinary, and CVC: central venous catheter.

in 2008 that was conducted in the same structure as ours (18\%). In general, Enterobacteriaceae isolated from the positive blood cultures, coming from the ICU during the period of study, showed a very high rate of resistance to thirdgeneration cephalosporins related essentially to an ESBL phenotype present in $42.5 \%$ of all isolated strains of Enterobacteriaceae and touching in the first isolates of Klebsiella pneumoniae. Pseudomonas aeruginosa was isolated from 9.1\% of bacteraemic episodes; this frequency joined the results of the literature $[1,10]$.

Staphylococcus aureus in this study represented $9.1 \%$ of bacteraemia. This rate is lower than the data reported by other studies [10] and this could be due to the low rate of catheter bloodstream infections in our study. MRSA rate was $20 \%$, which is lower than the results reported by several studies $[12,19]$. Among the Gram positive bacteria, we did not encounter any resistance to vancomycin or teicoplanin in our isolates; this result is completely different from several studies $[12,19]$. We have detected four cases of positive blood cultures for Candida, and only one corresponded to bacteraemia. A low rate of positive blood cultures yeast was also reported in the study of Elouennass et al. [21]. We can retain as an explanation the fact that blood cultures performed on conventional aerobic bottle, all the more on anaerobic environment, are less efficient than blood cultures performed on Sabouraud medium [22].

The absence of coagulase negative staphylococci (CNS) bacteraemia in our study can be explained by the fact that the judgment criterion for the definition of these infections requires the presence of several positive blood cultures with the same antibiotype, which was missing in our case and this could be unnoticed true CNS bacteraemia. On the other hand, the most threatening germ in contamination was CNS; Martinez et al. reported that $95 \%$ of germs contaminating blood cultures drawn from central lines and $88 \%$ of germs contaminating blood taken from peripheral vein blood cultures are CNS [23].

The problem of subjectivity in the interpretation of a positive blood culture certainly plays a role in the difference of the results observed in the literature and it is thus necessary to recall the difficulty of establishing the clinical significance of low virulence isolates such as commensal flora.

The most frequent source of bacteraemia microbiologically identified was the respiratory system (33\%) followed by urinary system (10\%). This is in agreement with many reports [15]. In cases where no source has been determined, we suppose that undiagnosed vascular catheter infections or bacterial translocation from the gastrointestinal tract may explain the infection. 
TABLE 3: Antimicrobial resistance rate of Gram negative bloodstream isolates.

\begin{tabular}{|c|c|c|c|c|c|}
\hline Drug & $\begin{array}{l}\text { Enterobacteriaceae } \\
\qquad(n=25)\end{array}$ & $\begin{array}{c}\text { Klebsiella } \\
\text { Pneumoniae } \\
(n=10)\end{array}$ & $\begin{array}{l}\text { Nonfermentative } \\
\text { GNB }(n=20)\end{array}$ & $\begin{array}{l}\text { Acinetobacter baumannii } \\
\qquad(n=13)\end{array}$ & $\begin{array}{c}\text { Pseudomonas } \\
\text { aeruginosa }(n=5)\end{array}$ \\
\hline Amoxi-Clavulanate & $64,2 \%$ & $60 \%$ & NT & NT & NT \\
\hline Pip-Tazobactam & $23,8 \%$ & $20 \%$ & $82,3 \%$ & $100 \%$ & $20 \%$ \\
\hline Cefalotin & $64,2 \%$ & $60 \%$ & NT & NT & NT \\
\hline Ceftazidime & $57,1 \%$ & $60 \%$ & $70 \%$ & $100 \%$ & $20 \%$ \\
\hline Ceftriaxone & $50 \%$ & $60 \%$ & NT & NT & NT \\
\hline Imipenem & $0 \%$ & $0 \%$ & $78,9 \%$ & $100 \%$ & $20 \%$ \\
\hline Ertapenem & $12,5 \%$ & $20 \%$ & NT & NT & NT \\
\hline Cefoxitime & $8,3 \%$ & $10 \%$ & NT & NT & NT \\
\hline Gentamicin & $37,5 \%$ & $50 \%$ & $63,1 \%$ & $83,3 \%$ & $20 \%$ \\
\hline Tobramycin & $32 \%$ & $50 \%$ & $50 \%$ & $53,8 \%$ & $20 \%$ \\
\hline Amikacin & $4 \%$ & $0 \%$ & $47,3 \%$ & $50 \%$ & $20 \%$ \\
\hline Netilmicin & $36 \%$ & $50 \%$ & $45 \%$ & $46,1 \%$ & $40 \%$ \\
\hline Ciprofloxacin & $40 \%$ & $70 \%$ & $70 \%$ & $100 \%$ & $20 \%$ \\
\hline Cotrimoxazole & $64 \%$ & $80 \%$ & $85 \%$ & $92,3 \%$ & $100 \%$ \\
\hline Fosfomycin & $0 \%$ & $0 \%$ & NT & NT & NT \\
\hline Colistin & $0 \%$ & $0 \%$ & $0 \%$ & $0 \%$ & $0 \%$ \\
\hline Ticarcillin & NT & NT & $88,2 \%$ & $100 \%$ & $33,3 \%$ \\
\hline Piperacillin & NT & NT & $72,2 \%$ & $100 \%$ & $20 \%$ \\
\hline Rifampicin & NT & NT & $42,8 \%$ & $46,1 \%$ & $0 \%$ \\
\hline
\end{tabular}

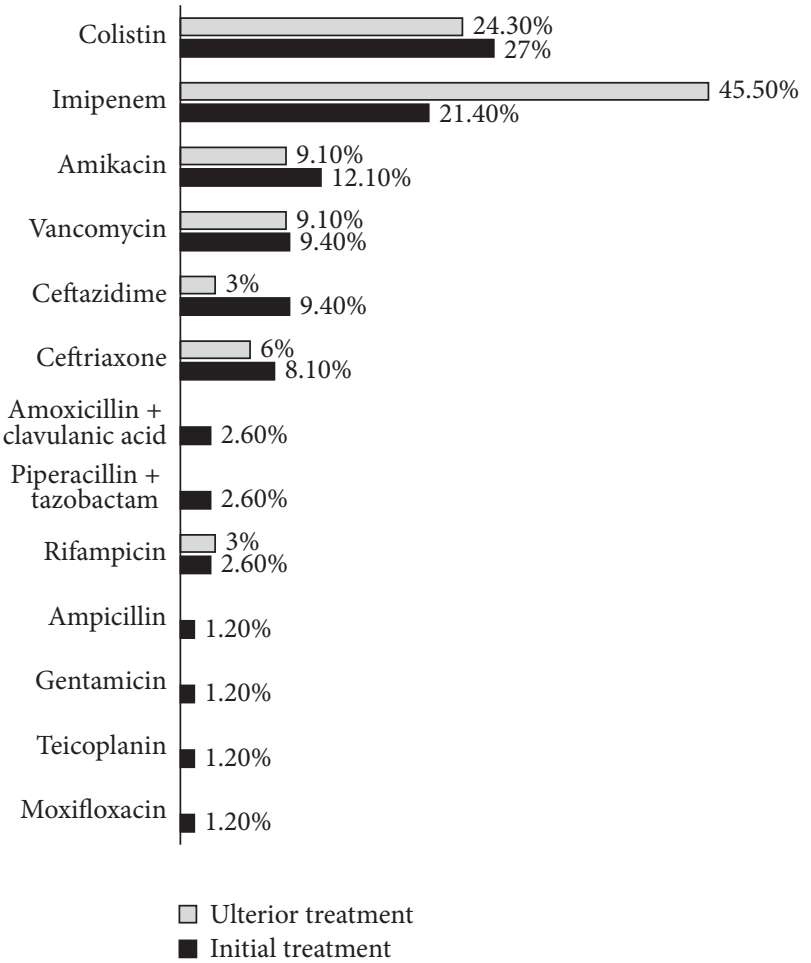

FIGURE 1: Molecules prescribed for initial and ulterior antibiotic therapy.

In terms of treatment, the initial treatment adequacy rate is the most discussed criterion in international journals because it influences the evolution of bacteraemic patients. Comparing our results to those in the literature, we noted that the adequacy rate of initial antibiotic therapy in our study is comparable to that reported by many studies $[1,10]$.

Colistin emerged in the last decade as a savior for the treatment of critically septic patients who suffer MDR-GNB infections [24]. It is frequently used as combination therapy in order to maximize killing effect of pathogens, reducing rate of resistance, optimizing clinical outcome, and reducing mortality [25]. However two earlier meta-analyses published in 2003 and 2004 in BMJ revealed that combination therapy did not change rates of fatality and increased adverse events [24].

In regard to our practice, colistin is usually used in combination with imipenem for the treatment of severe infections especially those due to Acinetobacter baumannii. It is used as monotherapy in the case of MDR Acinetobacter baumannii infections, taking into account the clinical condition and the evolution of the patient.

Pirson et al. studied the total cost of bacteraemia through a retrospective analysis of two cohorts totaling 1344 patients in a Belgian establishment in 2001. The authors concluded that bacteraemia was related to additional cost of $€ 12,853$ per patient [26].

According to this study, we can confirm the additional cost associated with bacteraemia in terms of consumption of antibiotics, but the extent of this problem cannot be measured because of the existence of socioeconomic differences preventing us from comparing our results to those of Western studies on one hand and the lack of studies of the cost in the developing countries on the other hand. 
TABLE 4: Characteristics of antimicrobial treatment.

\begin{tabular}{|c|c|c|c|c|}
\hline \multirow{2}{*}{ Characteristics } & \multicolumn{2}{|c|}{ Initial treatment $(n=43)$} & \multicolumn{2}{|c|}{ Further treatment $(n=17)$} \\
\hline & Number & $\%$ & Number & $\%$ \\
\hline \multicolumn{5}{|l|}{ (i) Delay of introduction: } \\
\hline (a) $<24 \mathrm{~h}$ & 42 & $98 \%$ & - & - \\
\hline (b) $24 \mathrm{~h} \ll 48 \mathrm{~h}$ & 0 & $0 \%$ & - & - \\
\hline (c) $>48 \mathrm{~h}$ & 1 & $2 \%$ & - & - \\
\hline \multicolumn{5}{|l|}{ (ii) Type of prescription: } \\
\hline (a) Not specifically adapted & 22 & $51 \%$ & - & - \\
\hline (b) Probabilistic & 20 & $46,5 \%$ & 7 & $41 \%$ \\
\hline (c) Documented & 1 & $2,5 \%$ & 10 & $59 \%$ \\
\hline \multicolumn{5}{|l|}{ (iii) Type of treatment: } \\
\hline (a) Monotherapy & 18 & $43 \%$ & 3 & $18 \%$ \\
\hline (b) Association & 25 & $57 \%$ & 14 & $82 \%$ \\
\hline (iv) Average length of initial treatment: (mean $+/-\mathrm{SD}$ ) & & & & \\
\hline \multicolumn{5}{|l|}{ (v) Adequacy of treatment: } \\
\hline (a) Adequate & 31 & $72 \%$ & 16 & 93 \\
\hline (b) Inadequate & 12 & $28 \%$ & 1 & $7 \%$ \\
\hline \multicolumn{5}{|l|}{ (vi) Effectiveness: } \\
\hline (a) Efficient & 27 & $63 \%$ & 6 & $35 \%$ \\
\hline (b) Ineffective & 16 & $37 \%$ & 11 & $65 \%$ \\
\hline \multicolumn{5}{|l|}{ (vii) Initial treatment modification reason: } \\
\hline (a) Escalation & - & - & 4 & $21 \%$ \\
\hline (b) Deescalation & - & - & 13 & $79 \%$ \\
\hline \multicolumn{5}{|l|}{ (viii) Most prescribed family: } \\
\hline (a) Beta-lactams & 12 & $28 \%$ & 3 & $18 \%$ \\
\hline (b) Polymyxins & 8 & $18,7 \%$ & - & - \\
\hline (c) Beta-lactams + aminoglycoside & 8 & $18,7 \%$ & 1 & $6 \%$ \\
\hline (d) Beta-lactams + polymyxins & 6 & $14 \%$ & 8 & $47 \%$ \\
\hline \multicolumn{5}{|l|}{ (ix) The most commonly used antibiotics: } \\
\hline (a) Imipenem & 9 & $21,4 \%$ & 8 & $45,5 \%$ \\
\hline (b) Colistin & 12 & $27 \%$ & 4 & $23,3 \%$ \\
\hline (c) Amikacin & 28 & $12,1 \%$ & 2 & $9,1 \%$ \\
\hline (d) Vancomycin & 22 & $9,4 \%$ & 2 & $9,1 \%$ \\
\hline \multicolumn{5}{|l|}{ (x) Cost of treatment: } \\
\hline (a) Total cost per patients & \multicolumn{4}{|c|}{ 899,27 Euros [259,85-1544,69] } \\
\hline (b) Daily cost per patients & \multicolumn{4}{|c|}{31,01 Euros $[17,48-54,59]$} \\
\hline
\end{tabular}

Our study shows a death rate of $69 \%$; this rate appears higher than those reported by several international studies $[1,10]$. The heterogeneity of the studied populations, the endemic rate of multiresistant bacteria, the management difficulties, and the differences among the work designs followed by each team could be explanations for this difference.

Mortality related to bacteraemic episodes increases with age, especially for patients over 75 years old who are frequently hospitalized, are suffering from several diseases, and have a reduced immune status. Furthermore clinical presentation of bacteraemia in this population is sometimes atypical, delaying diagnosis [27]. In our study, a difference of 10-year average was noted between patients who died and those who survived in favor of the first. But this result appears to be not statistically significant; this requires the recruitment of a larger number of patients in future studies. Nosocomial bloodstream infections are known to have a higher attributable mortality than infections acquired outside the hospital; Garrouste-Orgeas and colleagues [28] found that nosocomial bacteraemia was associated with a threefold increase in mortality. In our case, we were not able to draw such a conclusion, since we have a single episode bacteraemic community. Mortality due to episodes of bacteraemia increases with the accumulation of predisposing conditions in the same patient. Indeed, the identification of underlying illness in patients admitted to ICU darkens the 
TABLE 5: Comparison between the dead and survived patients in the study based on demographic, clinical, and microbiological profiles.

\begin{tabular}{|c|c|c|c|c|c|c|}
\hline \multirow{2}{*}{\multicolumn{2}{|c|}{ Variable }} & \multicolumn{2}{|c|}{ Survived $(n=12)$} & \multicolumn{2}{|c|}{ Dead $(n=27)$} & \multirow{2}{*}{ Signification } \\
\hline & & Number & $\%$ & Number & $\%$ & \\
\hline \multicolumn{2}{|c|}{ Age $($ mean +/-SD) } & \multicolumn{2}{|c|}{$52,1+/-20,1$} & \multicolumn{2}{|c|}{$62,3+/-12,5$} & NS \\
\hline \multirow{2}{*}{ Gender } & $\mathrm{M}$ & 4 & $33 \%$ & 11 & $40,7 \%$ & \multirow{2}{*}{ NS } \\
\hline & $\mathrm{F}$ & 8 & $67 \%$ & 16 & $59,2 \%$ & \\
\hline \multirow{5}{*}{ Underlying illness } & Diabetes mellitus & 3 & $25 \%$ & 9 & $33,3 \%$ & NS \\
\hline & Essential hypertension & 1 & $8,3 \%$ & 10 & $37 \%$ & NS \\
\hline & ID & 1 & $8,3 \%$ & 1 & $3,7 \%$ & NS \\
\hline & Chronic renal diseases & 0 & $0 \%$ & 2 & $7,4 \%$ & NS \\
\hline & Cardiopathy & 1 & $8,3 \%$ & 3 & $11,1 \%$ & NS \\
\hline \multirow{2}{*}{ Origin of bacteremia } & Nosocomial & 12 & $100 \%$ & 26 & $96,2 \%$ & NS \\
\hline & Ambulatory & 0 & $0 \%$ & 1 & $3,7 \%$ & NS \\
\hline \multirow{2}{*}{ Nature of bacteremia } & Primary & 7 & $58,3 \%$ & 15 & $55,5 \%$ & NS \\
\hline & Secondary & 5 & $41.6 \%$ & 12 & $44,4 \%$ & NS \\
\hline \multirow{4}{*}{ Source of bacteraemia } & Lungs & 3 & $25 \%$ & 10 & $37 \%$ & NS \\
\hline & Urinary tract & 2 & $16,6 \%$ & 2 & $7,4 \%$ & NS \\
\hline & CVC & 1 & $8,3 \%$ & 2 & $7,4 \%$ & NS \\
\hline & Unknown & 6 & $50 \%$ & 13 & $48,1 \%$ & NS \\
\hline \multirow{3}{*}{ Severity } & Sepsis & 9 & $75 \%$ & 7 & $25,9 \%$ & 0,007 \\
\hline & Severe sepsis & 2 & $16,6 \%$ & 4 & $14,8 \%$ & NS \\
\hline & Septic shock & 1 & $8,3 \%$ & 16 & $59,2 \%$ & 0,011 \\
\hline \multirow{3}{*}{ Type of stained bacteria } & GNB & 8 & $67 \%$ & 25 & $92,5 \%$ & NS \\
\hline & GPC & 3 & $25 \%$ & 2 & $7,4 \%$ & NS \\
\hline & MDR & 9 & $75 \%$ & 17 & $62,9 \%$ & NS \\
\hline \multirow{4}{*}{ Microorganism } & A. baumannii & 3 & $25 \%$ & 10 & $37 \%$ & NS \\
\hline & K. pneumoniae & 3 & $25 \%$ & 7 & $25,9 \%$ & NS \\
\hline & P. aeruginosa & 2 & $16,6 \%$ & 3 & $11,1 \%$ & NS \\
\hline & S. aureus & 3 & $25 \%$ & 2 & $7,4 \%$ & NS \\
\hline \multirow{2}{*}{ Adequacy of treatment } & Adequate & 5 & $41.6 \%$ & 18 & $66,6 \%$ & NS \\
\hline & Inadequate & 5 & $41.6 \%$ & 9 & $33,3 \%$ & NS \\
\hline
\end{tabular}

prognosis of bacteraemia [27]. The presence of an antecedent of hospitalization, corticosteroid therapy, antibiotic therapy, and surgery appear to be important risk factors of mortality. These criteria also stand out as mortality risk factors in the study of Kang et al. [29]. We observed a higher mortality rate when the source of infection was pulmonary; such observations are found in the literature [27]. Several studies have shown that mortality from bacteraemic episodes increases with inadequate treatment. Our numbers are too small to be interpreted. There are intuitively interests to start early empirical antibiotic that is active in vitro on the germ involved; this benefit has been demonstrated in several studies [30].

\section{Conclusions}

This study highlights the predominance of Gram negative bacteria and the emergence of multidrug-resistant organisms. Our results confirm some facts reported in many publications like the resistance to antibiotics and the attributable mortality; however, they differed in several important parameters like the clinical significance of positive blood cultures for CNS and the microbial ecology of bacteraemia. We also found that the rate of appropriate antibiotic treatment in the intensive care units of our structure is comparable to that reported by some international hospitals in big fame. The main limit of our study is the low number of patients; this instigates us to lead a multicentric study in order to provide a more complete picture of microbial ecology and resistance trends and to produce meaningful guidelines for bacteraemia prevention.

\section{Abbreviations}

ICU: Intensive care units

ESBL: $\quad$ Extended-spectrum beta-lactamases

APACHE II: Acute Physiology and Chronic Health Evaluation II

GNB: $\quad$ Gram negative bacilli

CNS: Coagulase negative staphylococci. 


\section{Conflicts of Interest}

The authors declare that there are no conflicts of interest regarding the publication of this paper.

\section{References}

[1] J. Vallés, E. Calbo, E. Anoro et al., "Bloodstream infections in adults: importance of healthcare-associated infections," Journal of Infection, vol. 56, no. 1, pp. 27-34, 2008.

[2] K. B. Laupland, "Incidence of bloodstream infection: a review of population-based studies," Clinical Microbiology and Infection, vol. 19, no. 6, pp. 492-500, 2013.

[3] A. W. Karchmer, "Nosocomial bloodstream infections: organisms, risk factors, and implications," Clinical Infectious Diseases, vol. 31, no. 4, pp. S139-S143, 2000.

[4] A.-S. Ouédraogo, A. Dakouré-Kissou, G. E. A. Poda, F. Koueta, D. Yé-Ouattara, and R. Ouédraogo-Traoré, "Epidemiology, microbiology, and outcomes of septicemia in children treated at the Charles de Gaulle University Pediatric Hospital in Burkina Faso," Santé, vol. 21, no. 4, pp. 221-225, 2011.

[5] T. J. Kirn and M. P. Weinstein, "Update on blood cultures: how to obtain, process, report, and interpret," Clinical Microbiology and Infection, vol. 19, no. 6, pp. 513-520, 2013.

[6] S. Balkaran-Amorim, Prise en charge des bactériémies dans le service des maladies du sang du Centre Hospitalier Régional et Universitaire de Lille en 2011 [Ph.D. thesis], 2012.

[7] Comité de l'Antibiogramme de la Société Française de Microbiologie (CASFM), Recommandations 2011, Société Française de Microbiologie, Paris, France, 2011, http://www .sfm-microbiologie.org.

[8] H. Seifert, "The Clinical importance of microbiological findings in the diagnosis and management of bloodstream infections," Clinical Infectious Diseases, vol. 48, no. 4, pp. S238-S245, 2009.

[9] S. Nielsen, "The incidence and prognosis of patients with bacteremia," Danish Medical Journal, vol. 62, no. 7, Article ID B5128, 2015.

[10] F. Y. Khan, S. S. Elshafie, M. Almaslamani et al., "Epidemiology of bacteraemia in Hamad general hospital, Qatar: a one year hospital-based study," Travel Medicine and Infectious Disease, vol. 8, no. 6, pp. 377-387, 2010.

[11] S. Hassoune, S. Nani, M. Ouhadous, O. Aalloula, M. Benbachir, and A. Maaroufi, "Incidence des bactériémies nosocomiales dans les services à haut risque du centre hospitalier universitaire de Casablanca (Maroc)," Pratiques et Organisation des Soins, vol. 43, no. 1, pp. 19-24, 2012.

[12] K. B. Laupland, H. Lee, D. B. Gregson, and B. J. Manns, "Cost of intensive care unit-acquired bloodstream infections," Journal of Hospital Infection, vol. 63, no. 2, pp. 124-132, 2006.

[13] X. Zhang, M.-M. Tong, M.-Z. Zhang, and H.-P. Zhu, "Risk factors of nosocomial bloodstream infections in surgical intensive care unit," International Journal of Clinical and Experimental Medicine, vol. 8, no. 9, pp. 16682-16687, 2015.

[14] A. Tabah, D. Koulenti, K. Laupland et al., "Characteristics and determinants of outcome of hospital-acquired bloodstream infections in intensive care units: The EUROBACT International Cohort Study," Intensive Care Medicine, vol. 38, no. 12, pp. 1930-1945, 2012.

[15] W. Sligl, G. Taylor, and P. G. Brindley, "Five years of nosocomial Gram-negative bacteremia in a general intensive care unit: epidemiology, antimicrobial susceptibility patterns, and outcomes,' International Journal of Infectious Diseases, vol. 10, no. 4, pp. 320-325, 2006.

[16] M. E. Stryjewski and H. W. Boucher, "Gram-negative bloodstream infections," International Journal of Antimicrobial Agents, vol. 34, pp. S21-S25, 2009.

[17] D. W. Wareham, D. C. Bean, P. Khanna et al., "Bloodstream infection due to Acinetobacter spp: epidemiology, risk factors and impact of multi-drug resistance," European Journal of Clinical Microbiology and Infectious Diseases, vol. 27, no. 7, pp. 607-612, 2008.

[18] R. Picot-Guéraud, P. Batailler, Y. Caspar, A. Hennebique, and M.-R. Mallaret, "Bacteremia caused by multidrug-resistant bacteria in a French university hospital center: 3 years of collection," American Journal of Infection Control, vol. 43, no. 9, pp. 960-964, 2015.

[19] C.-H. Su, J.-T. Wang, C. A. Hsiung et al., "Increase of carbapenem-resistant Acinetobacter baumannii infection in acute care hospitals in Taiwan: association with hospital antimicrobial usage," PLoS ONE, vol. 7, no. 5, Article ID e37788, 2012.

[20] T. A. Gulen, R. Guner, N. Celikbilek, S. Keske, and M. Tasyaran, "Clinical importance and cost of bacteremia caused by nosocomial multi drug resistant acinetobacter baumannii," International Journal of Infectious Diseases, vol. 38, pp. 32-35, 2015.

[21] M. Elouennass, I. Sahnoun, A. Zrara, T. Bajjou, and S. Elhamzaoui, "Epidemiology and susceptibility profile of blood culture isolates in an intensive care unit (2002-2005)," Medecine et Maladies Infectieuses, vol. 38, no. 1, pp. 18-24, 2008.

[22] L. L. Horvath, B. J. George, C. K. Murray, L. S. Harrison, and D. R. Hospenthal, "Direct comparison of the BACTEC 9240 and BacT/ALERT 3D automated blood culture systems for candida growth detection," Journal of Clinical Microbiology, vol. 42, no. 1, pp. 115-118, 2004.

[23] J. A. Martinez, J. A. DesJardin, M. Aronoff, S. Supran, S. A. Nasraway, and D. R. Snydman, "Clinical utility of blood cultures drawn from central venous or arterial catheters in critically ill surgical patients," Critical Care Medicine, vol. 30, no. 1, pp. 7-13, 2002.

[24] J. W. Al Ramahi, A. Al Bashaireh, and S. Salman, "Colistin in critically ill patients: a critical review," The International Arabic Journal of Antimicrobial Agents, vol. 4, no. 2, 2014.

[25] I. A. Bliziotis, G. Samonis, K. Z. Vardakas, S. Chrysanthopoulou, and M. E. Falagas, "Effect of aminoglycoside and $\beta$-lactam combination therapy versus $\beta$-lactam monotherapy on the emergence of antimicrobial resistance: a meta-analysis of randomized, controlled trials," Clinical Infectious Diseases, vol. 41, no. 2, pp. 149-158, 2005.

[26] http://www.senat.fr/rap/r05-421/r05-42113.html.

[27] V. Baudat, Hémocultures positives à l'Hôpital Cantonal de Fribourg, 1997-1998: signification clinique, microbiologie, épidémiologie, traitement et pronostic [Ph.D. thesis], University of Geneva, Geneva, Switzerland, 2002.

[28] M. Garrouste-Orgeas, J. F. Timsit, M. Tafflet et al., "Excess risk of death from intensive care unit-acquired nosocomial bloodstream infections: a reappraisal," Clinical Infectious Diseases, vol. 42, pp. 1118-1126, 2006.

[29] C.-I. Kang, S.-H. Kim, B. P. Wan et al., "Bloodstream infections caused by antibiotic-resistant gram-negative bacilli: risk factors for mortality and impact of inappropriate initial antimicrobial therapy on outcome," Antimicrobial Agents and Chemotherapy, vol. 49, no. 2, pp. 760-766, 2005. 
[30] A. Akpabie, C. Duché, and M. Le Gaudion, "Nosocomial bacteremia: impact of empirical antimicrobial treatment on the patients' outcome," Pathologie Biologie, vol. 57, no. 1, pp. 51-55, 2009. 


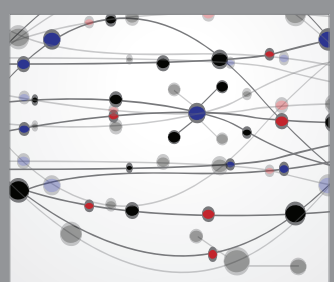

The Scientific World Journal
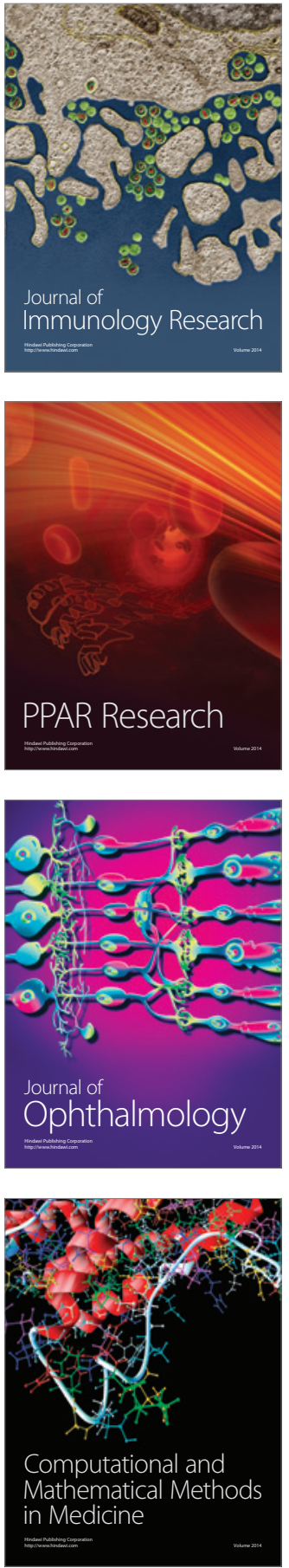

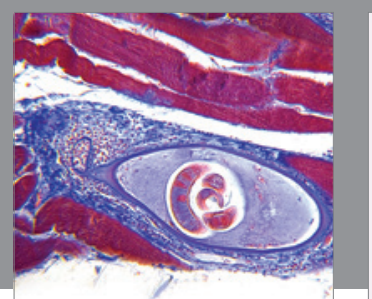

Gastroenterology Research and Practice
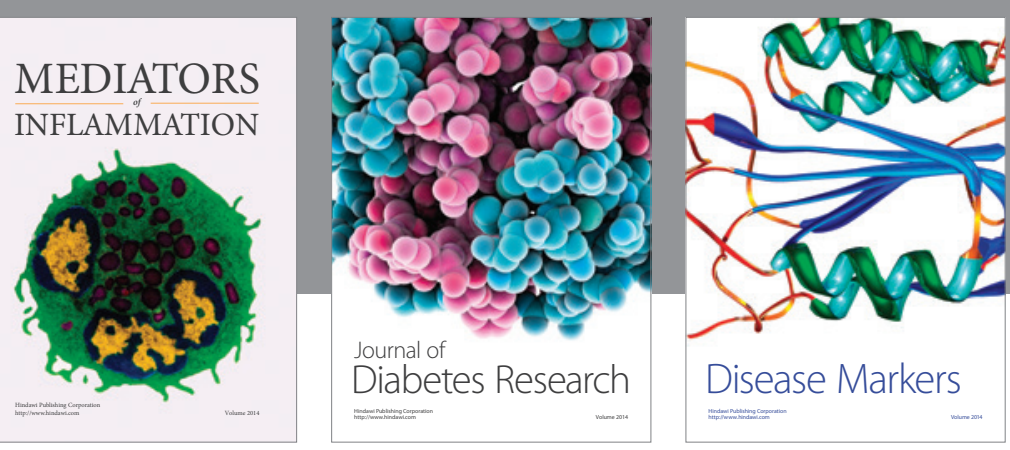

Disease Markers

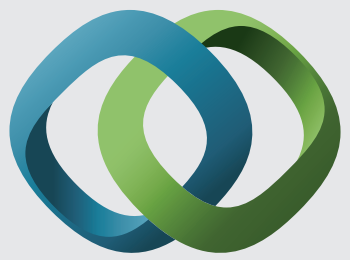

\section{Hindawi}

Submit your manuscripts at

https://www.hindawi.com
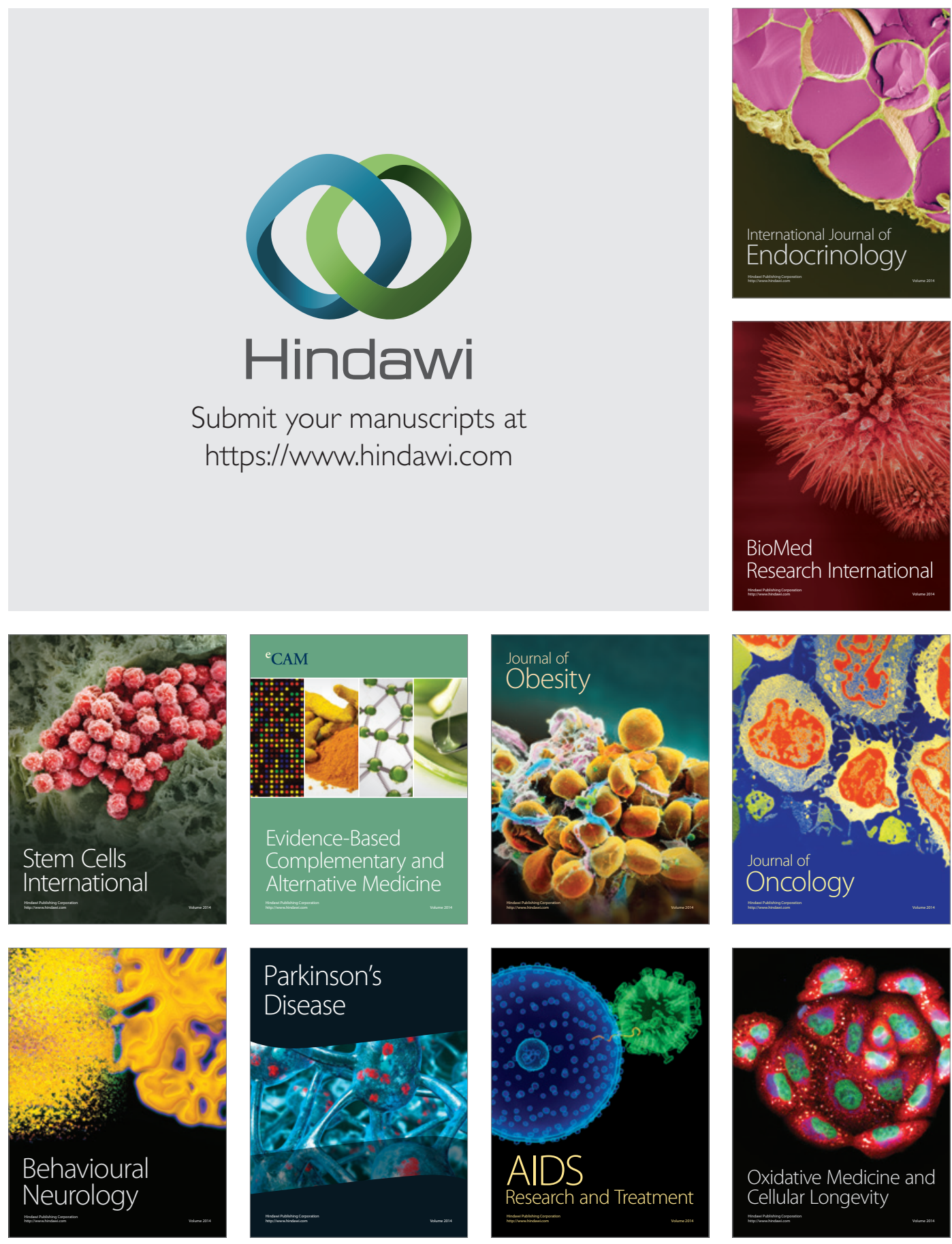